\title{
Self-assessment corner
}

\section{Sciatica}

\author{
A Jackson, CAJ Romanowski
}

\section{The patient}

A 58-year-old woman with a three-month history of left sciatica and claudication at 200 metres. Awaiting admission for coronary artery bypass graft for severe angina.

\section{Investigations}

Lumbar myelogram and computed tomography (CT) myelogram followed by left L4/5 facet joint CT arthrogram with intra-articular steroid injection (see figures 1 and 2).

\section{Question}

What is the most likely diagnosis?
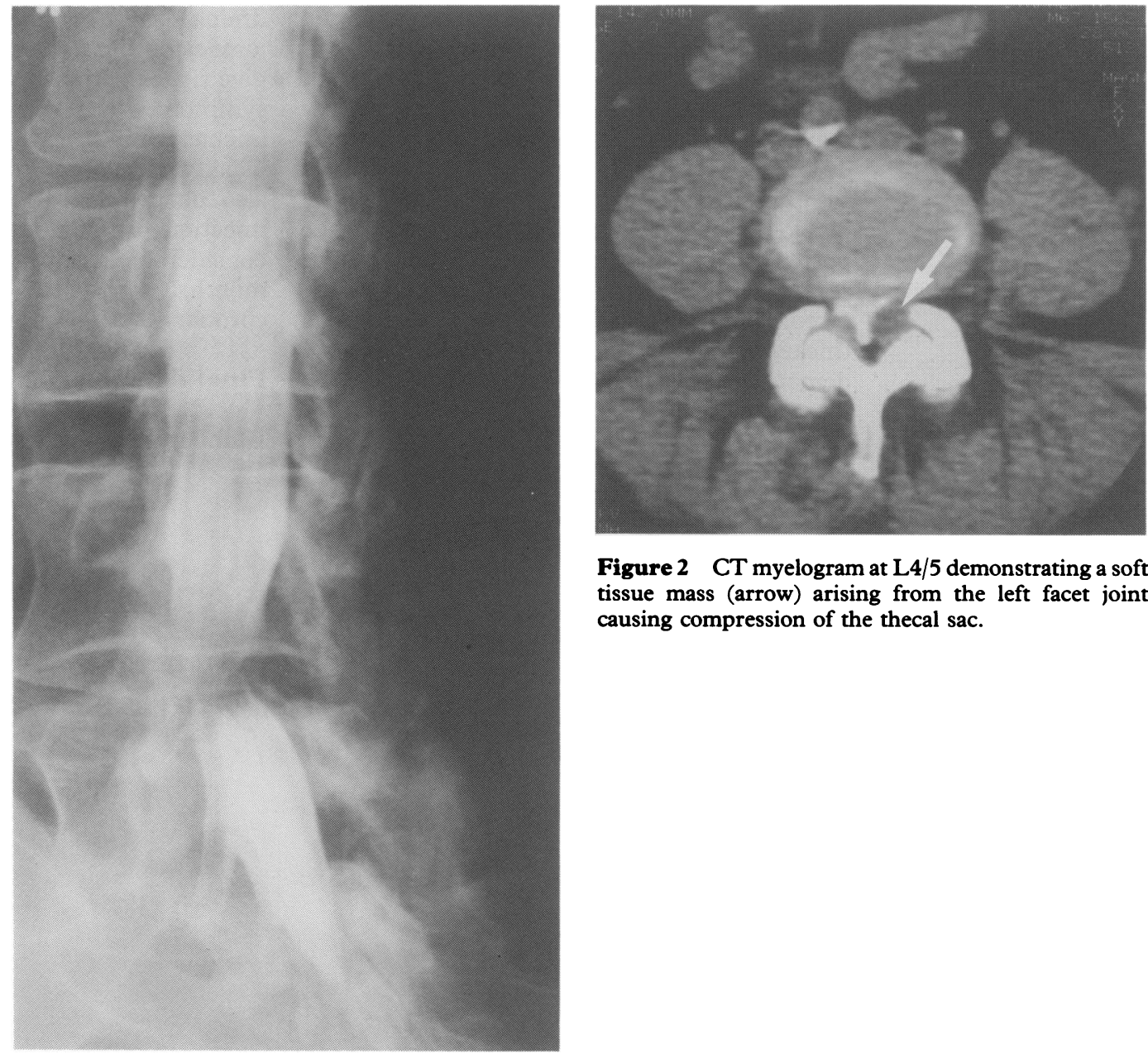

Figure 2 CT myelogram at L4/5 demonstrating a soft tissue mass (arrow) arising from the left facet joint causing compression of the thecal sac.
Figure 1 Lumbar myelogram showing severe spinal stenosis at $\mathrm{L} 4 / 5$ due to a left-sided posterior indentation of the thecal sac. 


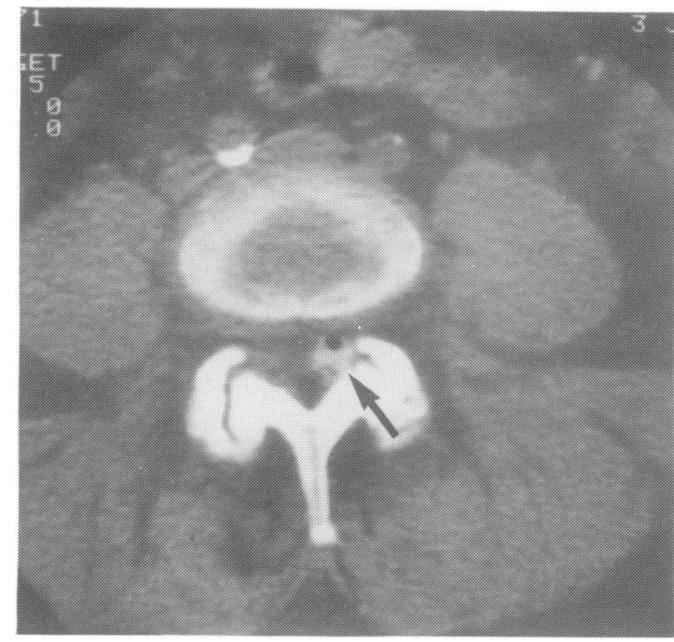

Figure 3 Post arthogram CT showing contrast and air within a synovial cyst (arrow).

\section{Answer}

Lumbar synovial cyst (figure 3).

\section{Comment}

Synovial cysts are a relatively common complication of osteoarthritis of the lumbar spine but only rarely reach a sufficient size to cause neural compression. Most symptomatic cases present with sciatica although approximately $20 \%$ have some evidence of spinal claudication, as in the current case. They are most common at the three lower lumbar disk levels and approximately $50 \%$ of symptomatic cases occur at L4/5. The myelographic findings are non-specific since posterior compression of the thecal sac can be seen with uncomplicated hypertrophic osteoarthropathy of the facet joint or with thickening of the ligamentum flavum. CT scan demonstrates a soft tissue mass arising from the facet joint which is usually of slightly higher attenuation than the thecal sac and which may

\section{Learning points}

- Synovial cysts of the lumbar facet joints may cause sciatica or spinal stenosis

- They are commonest at the lower three intervertebral joints

- They are suggested when the thecal sac is compressed from behind by a soft tissue mass on myelography or CT

- T2-weighted MRI demonstrate a high signal (fluid) centre to the cyst which enables confident diagnosis

- Facet joint arthrography is not required for diagnosis but intra-articular steroid injections can provide prolonged symptomatic relief

contain calcium in its wall. They occasionally contain air which arises in the degenerate facet joint and which may be seen on CT or even, rarely, on plain radiographs. With the increasing availability of magnetic resonance imaging (MRI) this is now the preferred method for investigation of sciatica. Synovial cysts are well seen on MRI where they demonstrate a central high signal on T2-weighted images due to their cystic nature. Since they communicate with the facet joint the diagnosis can be confirmed by facet joint arthrography although this is seldom required. Some synovial cysts will resolve following drainage and instillation of steroids into the joint cavity. In some cases steroid injection may give rise to permanent relief from neurological symptoms even though the cysts remain unchanged in size. Injection of local anaesthetic usually gives symptomatic relief which may also be prolonged. In the current case, the injection of lignocaine and steroids gave considerable symptomatic relief for over six months, whilst the patient recovered from coronary artery bypass grafts.

\section{Final diagnosis}

Lumbar synovial cyst. 